\title{
Analysis of the Performance of Neck Circumference to Identify Overweight and Obese Children
}

\author{
(D) Manuel André Virú-Loza \\ Hospital Nacional Edgardo Rebagliati Martins, Peru, South America
}

\section{Dear Editor,}

I have read with interest the article by Asif et al (1) on the potential usefulness of neck circumference to identify overweight and obese children. It is indeed a potential tool applicable to any context. However, there are two specific aspects about this article that I find interesting to point out.

In the statistical analysis section, they mention that a diagnostic test was considered highly accurate if the area under the curve (AUC) was between 0.65 and 1.00 , while it was moderately accurate if the AUC was between 0.5 and 0.65 (1). AUC values close to 0.5 are not useful in clinical practice so perhaps it would be better to refer to AUC values between 0.5 and 0.65 as "not accurate" rather than "moderately" accurate. That said, one could calculate the sample size required to find an AUC equal to the minimum considered "highly accurate", that is, 0.65. The pROC package (2) for $\mathrm{R}$ uses the formula published by Obuchowski et al (3) to perform this calculation. When using this package (using a power of 0.80 and a group ratio of $1: 1$ ) it is obtained that the required sample size is 110 (55 controls and 55 cases) for each AUC that is estimated to be at least 0.65 . In the study, four AUC values were calculated for each age group (overweight boys, overweight girls, obese boys and obese girls) (1). Therefore, for each age group, a minimum of 440 subjects would be required. One of the strengths of the study is that it far exceeds this number of subjects in each age subgroup (1). With the availability of such a large number of study subjects, it could have been considered to perform cross-validation, with which an internal validation of the AUCs could have been carried out.
However, for this, approximately 200 subjects would have been required as a minimum ideal number for each AUC to be validated (4). That is, a total of 800 subjects would have been required for each age group, which exceeds the number of subjects available to the researchers for several age groups (1). Despite this, similar to the way they grouped those aged 5 to 9 and 10 to 14 years (1), they could have made groups aged 5 to 6,7 to 8,9 to 10,11 to 12 and 13 to 14 years in order to have a sufficient number of subjects per group, not only to calculate cut-off points but also to be able to carry out an internal validation of the discriminative ability of neck circumference.

On the other hand, the group of 7-year-old boys had a very low AUC (0.555) unlike other groups analyzed (1). It would be important to propose possible explanations for this in order to know in which situations the neck circumference usefulness could be diminished.

\section{Ethics}

Peer-review: Internally peer-reviewed.

Financial Disclosure: The author declared that this study received no financial support.

\section{References}

1. Asif M, Aslam M, Wyszynska J, Altaf S, Ahmad S. Diagnostic performance of neck circumference and cut-off values for identifying overweight and obese pakistani children: a receiver operating characteristic analysis. J Clin Res Pediatr Endocrinol 2020;12:366-376.

2. Robin X, Turck N, Hainard A, Tiberti N, Lisacek F, Sanchez J, Müller M, Siegert S, Doering M. pROC: Display and Analyze ROC Curves v. 1.16.2. BMC Bioinformatics 2011;12:77 
Kontbay T and Turan I.

3. Obuchowski NA, Lieber ML, Wians FH Jr. ROC curves in clinical chemistry: uses, misuses, and possible solutions. Clin Chem 2004;50:1118-1125
4. Hastie T, Tibshirani R, Friedman J. Chapter 7: Model Assessment and Selection. In: Hastie T, Tibshirani R, Friedman J. The Elements of Statistical Learning: Data Mining, Inference, and Prediction. New York, Springer-Verlag, 2009;219-259. 